\title{
Variables mediadoras: ¿una oportunidad de profundizar en el estudio de la violencia desde el enfoque de la salud pública?
}

\author{
Mediating variables: an opportunity for in-depth \\ investigation of violence from a public \\ health perspective?
}

\author{
Variáveis mediadoras: uma oportunidade para \\ aprofundar o estudo da violência a partir da \\ perspectiva da saúde pública?
}

Chavez-Ayala et al. 1, al abordar la violencia, en especial la que se ejerce en los jóvenes y su migración, plantea dos asuntos de gran importancia no solamente de nuestro país, sino problemas compartidos con países hermanos de América Latina. Como lo señalan en la introducción de su trabajo, la relevancia de estos temas estriba tanto por el número importante de vidas que se cobran, como por la gran cantidad de variables que intervienen en su manifestación, lo que los hace complejos ya en sí mismos, tanto para su entendimiento como para la sugerencia de alternativas de solución.

El objetivo que planean en su trabajo Chavez-Ayala et al. ${ }^{1}$ es: "estimar la contribución de la exposición a la violencia en el hogar, el bullying y el ambiente violento escolar y violencia en el barrio con pensar o haber intentado emigrar a EE.UU. por los adolescentes mexicanos, con la intervención de variables de salud mental como la autoestima emocional y autoestima escolar, depresión y la ideación y el intento suicida, como mediadoras del efecto, para identificar los contextos sociales de riesgo y las formas de violencia, a fin de planificar estrategias y políticas de salud pública para la prevención de la migración internacional ilegal de los adolescentes".

Un aporte importante que hacen Chavez-Ayala et al. en este trabajo es conjugar dos problemas complejos (violencia y migración), a través de considerar variables mediadoras para explicar la conexión que tienen ambos. Pensando este planteamiento, propongo dos reflexiones:

\section{¿Es preciso partir del enfoque de la salud pública para estudiar la violencia en los adolescentes?}

Como lo mencionan en su trabajo Chavez-Ayala et al., la Organización Mundial de la Salud define la violencia como "el uso deliberado de la fuerza física o el poder, ya sea en grado de amenaza o efectivo contra uno mismo, otra persona, o contra un grupo o comunidad, que cause o tenga muchas probabilidades de causar lesiones, muerte, daños psicológicos, trastornos del desarrollo o privaciones”. En esta concepción, la violencia se establece como un acto y no como un hecho social.

Con este precepto trabajamos la violencia desde el enfoque de la salud pública, insuficiente en muchos casos para aproximarse a la complejidad del problema. Comparto lo que plantea Pellegrini Filho 2 cuando dice que: "Hablar de la 'aplicación' del enfoque de la salud pública (es decir, del enfoque de riesgo) a la violencia no sólo es inadecuado, porque implica una identificación forzada de la violencia, como si fuese una enfermedad, sino también porque supone considerar el enfoque de la salud pública como algo inmutable que
1 Facultad de Medicina, Universidad Nacional Autónoma de México, México DF, México.

\section{Correspondencia}

M. G. Alvear-Galindo Departamento de Salud Pública, Facultad de Medicina, Universidad Nacional Autónoma de México.

Ciudad Universitaria. Circuito Interior, Facultad de Medicina. México / DF 4530, México.

alvear@unam.mx 
se aplica a objetos distintos, que, a la postre, acaban reduciéndose a uno solo". En su trabajo Chavez-Ayala et al., al plantear variables mediadoras del efecto a variables de la salud mental, y considerar a estas como las causas que explican por qué migran los adolescentes, le suprimen a la violencia su condición de enfermedad.

No obstante, abordar la violencia desde el enfoque de la salud pública es identificarlo "con el enfoque de riesgo, según el cual los factores determinantes de carácter sociocultural suelen analizarse a partir de unas pocas variables, tratadas como si fueran atributos de individuos y no de grupos" 2 (p. 220), por lo que es indispensable estudiar a la violencia desde las particularidades de las sociedades y no a partir de las características de los individuos.

Desde el enfoque de la salud pública, se definen distintos tipos de violencia, como uno de ellos está la considerada como "violencia juvenil"; sin embargo, entenderla desde ahí no facilita la comprensión de la violencia que se ejerce hacia y entre los jóvenes; nos ayuda a cuantificar la violencia directa ejercida sobre los jóvenes, pero no a explicar su presencia desde el contexto social donde viven.

\section{¿Por qué violencia juvenil y no violencia estructural para entender la migración juvenil?}

Desde el enfoque de la salud pública, cuando un joven presenta conductas violentas su tratamiento estará dado por sus antecedentes biológicos, familiares y por las características del medio ambiente que lo rodea. Dependerá de su capacidad individual de negociar con su entorno e integrarse a él con una conducta normal/no violenta, lo que le permitirá relacionarse con sus pares y con su entorno social. De no ser así, el tratamiento individual y aislado será dado por las instituciones de control social (penal), las cuales intervienen sobre las personas y no sobre las situaciones.

Estudiar el problema de la violencia bajo el enfoque de la salud pública, y explicarla desde el modelo ecológico, contribuye a conocerla desde su expresión. Abordar el problema a partir de características individuales tiene alcances distintos que si se considera el problema a nivel poblacional, por lo que es preciso pensar en otras formas de aproximarse al problema de la violencia desde la salud pública.

Cuando hablamos de la violencia en el grupo de los adolescentes o jóvenes, el término de violencia estructural permite comprenderla con otra mirada. Siguiendo las ideas de La Parra \& Tortosa 3 (p. 57) como: "aquellas situaciones en las que se produce un daño en la satisfacción de las necesidades humanas básicas (supervivencia, bienestar, identidad o libertad) como resultado de los procesos de estratificación social, es decir, sin necesidad de formas de violencia directa”.

El desarrollo de la juventud se da en un contexto en donde "el repliegue del Estado benefactor, la fuerza creciente del mercado, la irrupción de los medios de comunicación, el descrédito de las instituciones y actores tradicionales (partidos, iglesias, sindicatos), la globalización, la migración, la fuerza del narcotráfico y del crimen organizado, constituyen no solamente un escenario, sino un entramado complejo, sistémico, multidimensional" 4 (p. 49). Con este panorama, donde los derechos, las capacidades y las libertades parecen no existir; la población juvenil se convierte en receptora de violencia estructural.

Bajo esta perspectiva, la violencia estructural participa en el proceso de exclusión de grandes sectores de la población, los sitúa en un lugar desde el cual no tienen posibilidades de acceder a los bienes sociales y culturales que ofrece el sistema social. La mayoría de los migrantes que van a los Estados Unidos, provenientes de México y países Centroamericanos, tienen como paso natural territorio mexicano. Siendo población indocumentada, los migrantes no pueden ser identificados, tienen condiciones de vida y de salud precarias, las expectativas que tienen para migrar son mejores condiciones de vida y trabajo. La migración de los jóvenes la podemos ver como la "búsqueda" de la tierra prometida.

Desde la violencia estructural, las variables mediadoras para explicar la migración de los adolescentes son aquellas que intervienen en la participación/inclusión de los jóvenes en los espacios tradicionales -escuela, trabajo y participación política institucional-, son estos elementos los que ayudan a explicar su migración, vista esta como la exclusión/despojo del lugar de origen.

La violencia es un problema complejo, invariablemente se aborda desde una posición política e ideológica. De acuerdo con el pensamiento de Butler 5 (p. 16) cuando afirma que: "no hay un solo marco y los marcos no son precisamente estáticos (...) el marco no simplemente contiene o exhibe lo que contiene, sino que participa activamente en una estrategia de contención, produciendo y haciendo cumplir de un modo selectivo lo que se contará como realidad". Bajo esta idea, entender la violencia en los jóvenes desde la violencia estructural, y no desde violencia juvenil, es otra forma de plantearla como problema de salud pública. 
Los resultados obtenidos del abordaje de la violencia a través del enfoque de la salud pública son muy desalentadores; se vuelve evidente la necesidad de reformular esta problemática y construir nuevas miradas para su estudio. El trabajo de Chavez-Ayala et al., contribuye a entender la violencia no como una enfermedad, sino como la generadora de ella. Al considerar variables mediadoras para comprender problemas complejos, como lo son la violencia en adolescentes y las causas de su migración, Chavez-Ayala et al. abren la posibilidad de generar nuevas explicaciones que permitan visualizar los problemas de salud pública desde diferentes marcos conceptuales.

1. Chavez-Ayala R, Orozco-Núñez E, Sánchez-Estrada M, Hernández-Girón C. Violencia y salud mental asociados a pensar o haber intentado emigrar internacionalmente por adolescentes mexicanos. Cad Saúde Pública 2017; 33:e00119516.

2. Pellegrini Filho A. La violencia y la salud pública. Rev Panam Salud Pública 1999; 5:219-21.

3. La Parra D, Tortosa JM. Violencia estructural: una ilustración del concepto. Documentación Social 2003; (131):57-72.
4. Reguillo R. La performatividad de las culturas juveniles. Estudios de Juventud 2004; (64):49-56.

5. Butler J. Violencia de estado, guerra, resistencia. Por una nueva política de la izquierda. "Las categorías nos dicen más sobre la necesidad de categorizar los cuerpos que sobre los cuerpos mismos" (entrevista de Daniel Gamper Sachse). Buenos Aires: Katz Editores/Barcelona: Centro de Cultura Contemporánea de Barcelona; 2011. 